\title{
OPTICAL AND STRUCTURAL PROPERTIES OF OPTICAL THIN FFILMS WITH VARIOUS COLUMNAR MICROSTRUCTURES
}

\author{
Seouk-Hoon Woo, Yong Jun Park, and Chang Kwon Hwangbo \\ Department of Physics, Inha University, Incheon 402-751, Republic of Korea
}

Received 27 November 2006

\begin{abstract}
In this study, optical and structural properties of the thin films prepared using glancing angle deposition (GLAD) are investigated. Various glancing angles and substrate rotations are employed to control the columnar microstructure of the films. The results show that as the glancing angle increases, the column angle and the porosity of $\mathrm{TiO}_{2}$ films increase and the refractive index decreases, due to shadow effects. The optical anisotropy of tilted and zigzag microstructure $\mathrm{TiO}_{2}$ films have a large anisotropy and reaches a maximum at a glancing angle of $60^{\circ}$, while that of helical microstructure $\mathrm{TiO}_{2}$ film deposited at $60^{\circ}$ with substrate rotation shows a very small anisotropy due to the symmetric structure. It is found that the optical anisotropy of $\mathrm{TiO}_{2}$ films deposited by GLAD can be controlled by modification of microstructure.
\end{abstract}

PACS: 81.15, 78. 20. Ek, 79. 70. +q

Keywords: $\mathrm{TiO}_{2}$ film, glancing angle deposition, microstructure, and optical anisotropy

\section{INTRODUCTION}

In general, optical thin films deposited at low substrate temperature reveal a columnar microstructure, due to low mobility and shadow effects: the columns are grown from the substrate to the top surface of the films and voids exist between the columns [1]. Therefore the optical and structural properties of the columnar thin films are inferior to the corresponding bulk materials. In the past, substantial efforts such as ion-beam assisted deposition, ion-beam sputtering, and so on, have been used to destroy the columnar microstructure and improve the density of the films [2 - 4].

However, minimal research has been carried out for applying a columnar microstructure for new optical coatings and devices [5 - 7]. Recently, modification of columnar microstructure using the glancing angle deposition (GLAD) technique has drawn attention because many applications require the nano-engineering technology of optical thin films. The GLAD technique is an oblique angle deposition of thin films to control the nano-scale structure of the films [8]. Obliquely deposited films exhibit anisotropic behaviors in optical and structural properties, in contrast to normally deposited films.

A schematic diagram of the glancing angle deposition with substrate rotation is presented in Fig. 1 (a). Young and Kowal [9] and later by others [10, 11], first reported the depositions at oblique incident angles. The columnar direction of these films is obliquely demonstrated in Fig. 1 (b) due to the shadow effects, and the column angle $\beta$ measured with respect to the substrate normal is smaller than the glancing angle, $\alpha$, between the flux of vapor and substrate normal 
$[11,12]$. The $\mathrm{x}$-direction is parallel to the substrate surface, the $\mathrm{y}$-direction is perpendicular to the vapor flux, and the $\mathrm{z}$-direction is normal to the substrate surface.

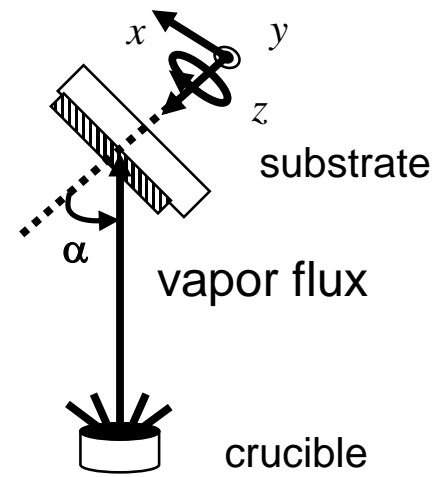

(a)

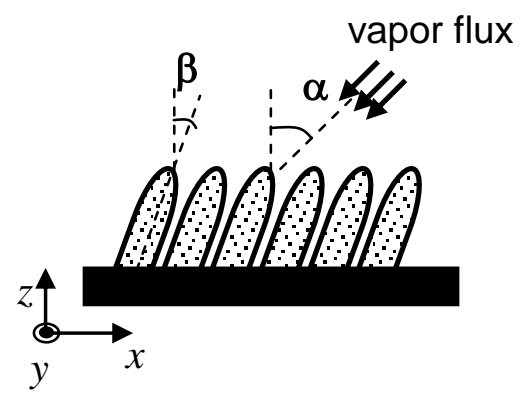

(b)

Fig. 1: (a) Schematic diagram of the glancing angle deposition with substrate rotation, (b) vapor flux, column, and film orientation with angle definitions

It is well known that GLAD is a technique for fabricating thin film materials with controlled microstructure [11 - 13]. This technique is based on physical vapor deposition and employs oblique angle deposition flux and substrate motion, to allow nanometer scale control of the structure in engineered thin film materials $[5,6]$. These obliquely deposited films exhibit a physical behavior different from normally deposited films and are used extensively for achieving desirable anisotropic properties for electrical, magnetic, and optical applications [14 18]. Optical anisotropic thin films are of interest because they can be used as optical components that cannot be constructed from isotropic layers. The optical anisotropy of the films can have various optical applications such as optical retardation plates [14], anisotropic antireflection coatings [17], birefrigent omnidirectional reflectors [18], three-dimensional photonic band gap crystals [19], and so on. In general, the anisotropic properties of these films originate from the microstructure, due to shadow effects [5, 6]. Therefore, it is important to investigate the relationship between optical anisotropy and columnar microstructure in optical thin films deposited by GLAD. Furthermore, it is expected that GLAD may be employed to control the microstructure and therefore control the optical anisotropy of the films.

This study presents the modification of the microstructure and optical properties of $\mathrm{TiO}_{2}$ film prepared by electron-beam evaporation using the GLAD technique. Various glancing angles and substrate rotation are employed in order to control the columnar microstructure of the films. The refractive index, optical anisotropy, microstructure, and surface morphologies of the films were investigated using a spectrophotometer, scanning electron microscopy (SEM), and variable angle spectroscopic ellipsometer (VASE). The relationship between the optical anisotropy and columnar microstructure in $\mathrm{TiO}_{2}$ films deposited by GLAD and the effects of the optical anisotropy on the microstructure, are also investigated.

\section{EXPERIMENTS}

$\mathrm{TiO}_{2}$ films were prepared by electron-beam evaporation using the GLAD technique. The deposition was performed in a vacuum chamber at a base pressure of $\sim 5 \times 10^{-6}$ Torr. The electron-beam evaporator with a $3 \mathrm{~cm}$ crucible pocket was located at $45 \mathrm{~cm}$, directly beneath the substrate. The deposition rate and the thickness of the growing films were measured by a quartzcrystal sensor, which was placed near the substrate. Glasses and $\mathrm{Si}$ wafers were used as substrates. Various glancing angles $\left(0^{\circ}, 30^{\circ}, 45^{\circ}, 60^{\circ}, 70^{\circ}\right.$, and $\left.80^{\circ}\right)$ and substrate rotation $(0.33$ 154 
rpm) were employed to control the microstructure of the GLAD films as tilted, zigzag, and helical structures. The deposition rate of the $\mathrm{TiO}_{2}$ films was kept at $0.3 \mathrm{~nm} / \mathrm{s}$, using a quartzcrystal sensor.

$\mathrm{TiO}_{2}$ films deposited on glass substrates were used for optical analysis, and films deposited on silicon wafers were used for structural analysis. Optical transmittance and reflectance of the films on glass substrates were measured by a spectrophotometer (Cary 500, Varian) in the wavelength range of 400 to $800 \mathrm{~nm}$. Polarizers were used to measure the transmittance and reflectance. The measurement of transmittance and reflectance was performed in two mutually orthogonal directions with a rotating linear polarizer: the $\mathrm{x}$-direction is parallel to the projection of vapor beam direction on the substrate surface, and the $\mathrm{y}$-direction is perpendicular to the $\mathrm{x}$ direction, as shown in Fig. 1 (b). The refractive index of the films was determined using an envelope method. The transmission intensity of the films as a function of rotation angle of the films between the two cross-polarizers was measured by a variable-angle spectroscopic ellipsometer (VASE). The cross-section and surface morphologies of the films were investigated using scanning electron microscopy (SEM).

\section{RESULTS AND DISCUSSION}

\subsection{Tilted structure $\mathrm{TiO}_{2}$ films}

The cross-sectional morphologies of tilted structure $\mathrm{TiO}_{2}$ films at $\alpha=0^{\circ}, 45^{\circ}, 60^{\circ}$ and $80^{\circ}$ in Fig. 2 show that the $\mathrm{TiO}_{2}$ films by GLAD have a tilted columnar microstructure. The column angles $(\beta)$ of the films with various glancing angles were obtained from the cross-sectional SEM micrographs using an image analysis method. It shows that the column angles $(\beta)$ and the intercolumnar gap (void) of the $\mathrm{TiO}_{2}$ films increase due to shadow effects, as the glancing angle increases.
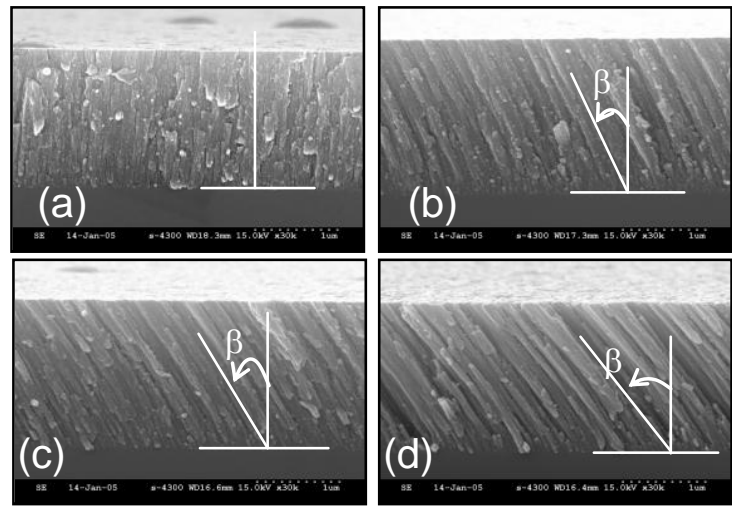

Fig. 2: Cross-sectional SEM images of tilted structure $\mathrm{TiO}_{2}$ films at various glancing angles: (a) $\alpha=0^{\circ}$, (b) $\alpha=45^{\circ}$, (c) $\alpha=60^{\circ}$, and (d) $\alpha=80^{\circ}$

The column angle of the tilted structure $\mathrm{TiO}_{2}$ films at various glancing angles is presented in Fig. 3. It is well known that the column angle $\beta$ is smaller than the vapor incident angle $\alpha$, following the empirical tangent rule, $\tan \beta=(1 / 2) \tan \alpha[20]$. However, the column angle $\beta$ of the $\mathrm{TiO}_{2}$ films in our experiments is not consistent with the tangent rule, as shown in Fig. 3 . Therefore, a modified tangent rule for $\mathrm{TiO}_{2}$ films is empirically proposed as follows;

$$
\tan \frac{\beta}{C}=\frac{1}{2} \tan \alpha
$$


where $C$ is a coefficient that may change with deposition conditions and materials. The coefficient $C=0.53$ for the $\mathrm{TiO}_{2}$ films is found to be much lower than when $C=1.0$ for the tangent rule. This result shows that the micro-column angle of the films can be controlled using the glancing angle.

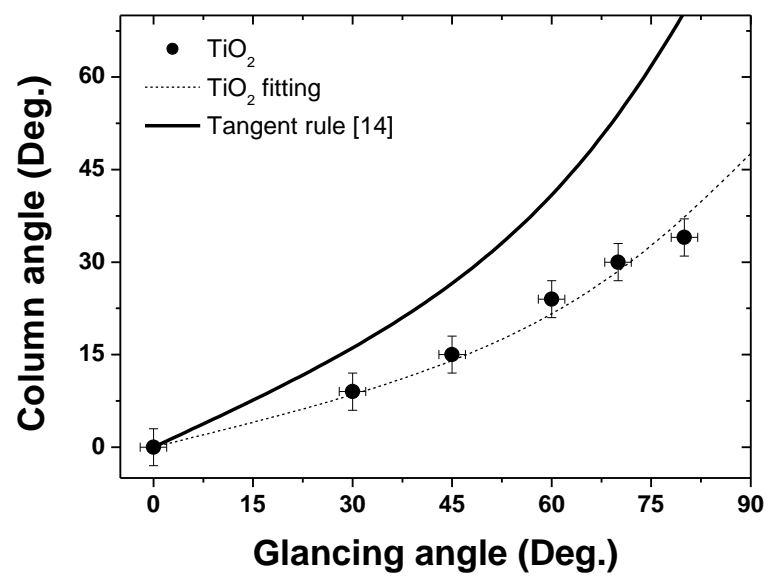

Fig. 3: Relationship between the column and glancing angles for $\mathrm{TiO}_{2}$ films

In order to investigate the anisotropic refractive index of $\mathrm{TiO}_{2}$ films, the transmittance was measured by a linear polarized light, in two orthogonal directions: $T_{x}$ in the x-direction parallel to the projection direction of the vapor flux, and $T_{y}$ in the y-direction. It is found that the transmittance in the $\mathrm{x}$-direction is different from that in the y-direction, at various oblique glancing angles. Then, refractive indices $n_{x}$ and $n_{y}$ of the $\mathrm{TiO}_{2}$ films are determined using an envelope method, from $T_{x}$ and $T_{y}$, respectively [1]. The result shows that $n_{x}$ of $\mathrm{TiO}_{2}$ films at $532 \mathrm{~nm}$ decreases drastically from 2.043 to 1.545 as $\alpha \square$ increases from $0^{\circ}$ to $80^{\circ}$. Similarly $n_{y}$ decreases from 2.043 to 1.580, as shown in Fig. 4(a).

The packing density $p$ of a film can be obtained using an approximate linear equation, $n_{f}=p n_{b}+(1-p) n_{v}$. Where $n_{b}$ is the refractive index of the bulk, $n_{f}$ is the mean refractive index of the film, and $n_{v}$ is the refractive index of the void (air, $n_{v}=1.0$ ). Therefore, the porosity can be given by $(1-p)$. As the glancing angle increases from $0^{\circ}$ to $80^{\circ}$, it is found that the mean porosity in the $\mathrm{x}$ - and y-direction of the $\mathrm{TiO}_{2}$ films increases from 20 to $58 \%$. The porosity in the $\mathrm{x}$-direction is larger than that in the y-direction, because the shadow effect of the $\mathrm{x}$-direction is much larger than that of the y-direction, due to asymmetric vapor flux, as shown in Fig. 1(b). In addition, this result is consistent with an increase in the inter-columnar gap (void) and a decrease in the refractive index of the $\mathrm{TiO}_{2}$ films as the glancing angle increases. 

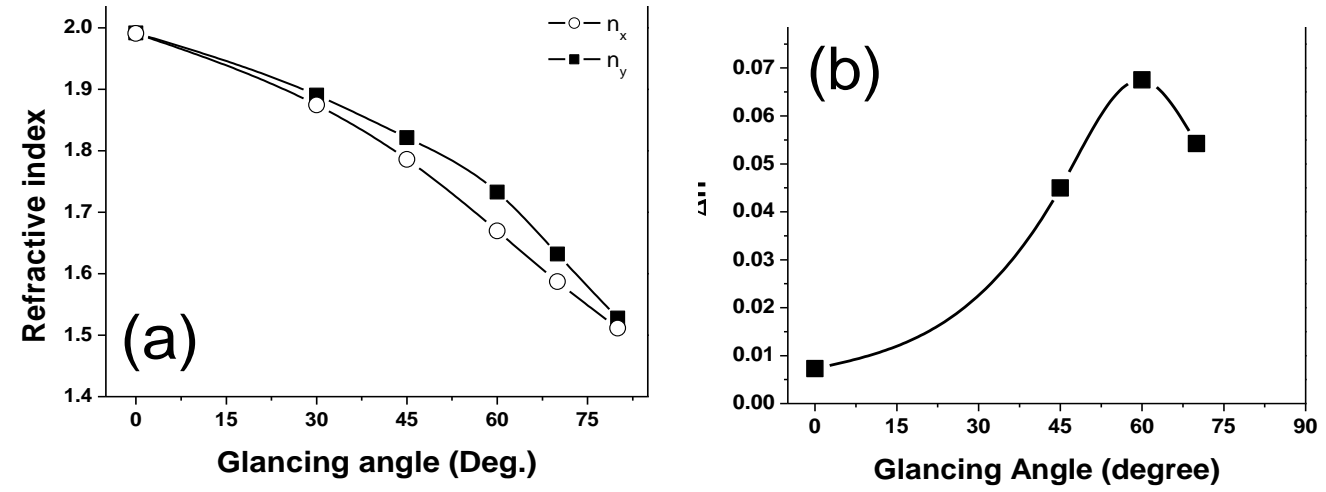

Fig. 4: Refractive index and optical anisotropy $(\Delta n)$ of tilted structure $\mathrm{TiO}_{2}$ films at various glancing angles: (a) Refractive index, and (b) optical anisotropy

The optical anisotropy is defined as $\Delta n=n_{y}-n_{x}$, which is the difference in refractive indices between $\mathrm{x}$ - and $\mathrm{y}$-directions. As glancing angle increases, the optical anisotropy $(\Delta n)$ of $\mathrm{TiO}_{2}$ films at $532 \mathrm{~nm}$ increases, and reaches the maximum value at $\alpha=60^{\circ}$, and then decreases, as shown in Fig. 4 (b). The $\mathrm{TiO}_{2}$ films deposited by GLAD with tilted structure have a large optical anisotropy $\Delta n=0.065$ at $532 \mathrm{~nm}$, which is consistent with previous reports $[14,16]$.

\subsection{Microstructure controlled $\mathrm{TiO}_{2}$ films}

Since the optical anisotropy of the tilted columnar structure is maximum at $\alpha \square=60^{\circ}$, the other structures such as zigzag and helical structures become of interest. The zigzag structure was deposited by flip-flopping the substrate at $\alpha \square=+60^{\circ}$, and $\alpha \square=-60^{\circ}$, and the helical structure was deposited by rotating the substrate at $0.33 \mathrm{rpm}$ at $\alpha \square=60^{\circ}$. The thicknesses of the $\mathrm{TiO}_{2}$ films for various structures were maintained at $730 \mathrm{~nm}$. The cross-section images of $\mathrm{TiO}_{2}$ films with tilted, zigzag, and helical structures are presented in Fig. 5 (a), (b), and (c).
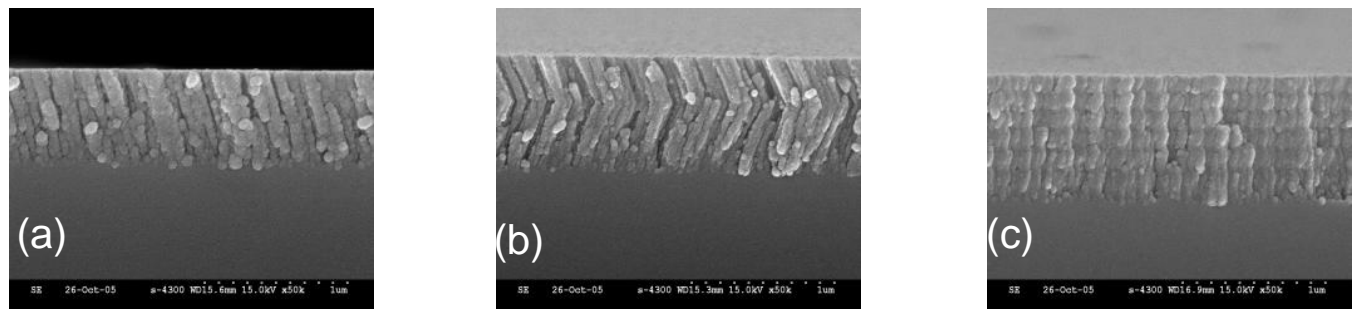

Fig. 5: Cross-sectional SEM images of $\mathrm{TiO}_{2}$ films at $\alpha=60^{\circ}$ : (a) tilted structure, (b) zigzag structure, and (c) helical structure

The column direction of the films with tilted and zigzag structure is oblique to the substrate in Fig. 5 (a) and (b), while the center axis of the film with helical structure is normal to the substrate in Fig. 5 (c). It appears that the helical structure consists of a series of disks due to the fast rotation of the substrate: the diameter of the disk of the $\mathrm{TiO}_{2}$ film with helical structure is approximately $80 \mathrm{~nm}$ and the pitch is approximately $120 \mathrm{~nm}$. The column diameter of the $\mathrm{TiO}_{2}$ films with tilted and zigzag structure is approximately $80 \mathrm{~nm}$. It shows that the glancing angle and substrate rotation are very useful for control of columnar microstructure. 


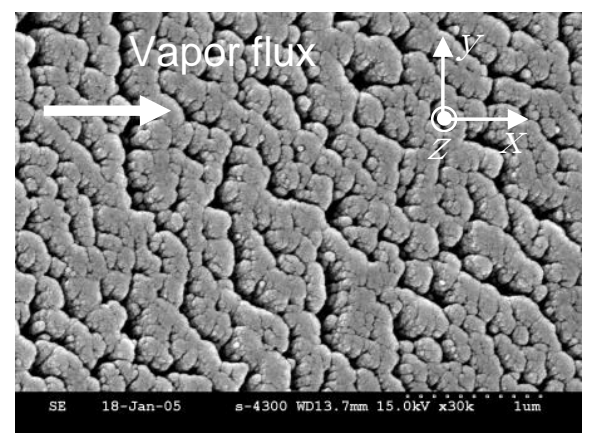

(a)

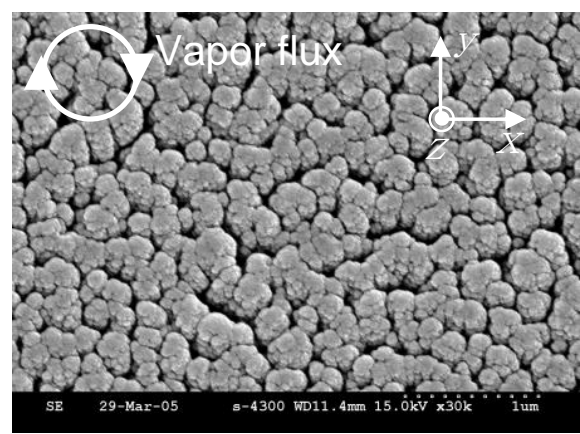

(b)

Fig. 6: Surface morphology of $\mathrm{TiO}_{2}$ films at $\alpha=60^{\circ}$ : (a) tilted and zigzag structure and (b) helical structure

However, circle-like shapes are observed in the top surface of the $\mathrm{TiO}_{2}$ film with a helical structure at $\alpha=60^{\circ}$ in Fig. 6 (b). The surface morphology of the $\mathrm{TiO}_{2}$ film with helical structure at $\alpha=60^{\circ}$ appears to be symmetric in the $\mathrm{x}$ - and $\mathrm{y}$-direction due to the symmetric incoming vapor flux. It is expected that the optical property of $\mathrm{TiO}_{2}$ film with a helical structure is isotropic, due to symmetric microstructure.

The optical anisotropy of the various columnar microstructure $\mathrm{TiO}_{2}$ films at $532 \mathrm{~nm}$ is presented in Fig. 7. The optical anisotropy $(\Delta n)$ of the $\mathrm{TiO}_{2}$ films with both tilted and zigzag structures at $532 \mathrm{~nm}$ is $\Delta n=0.065$, while the $\mathrm{TiO}_{2}$ film with helical structure shows a very small optical anisotropy, $\Delta n=0.004$. This result shows that the optical anisotropy of the $\mathrm{TiO}_{2}$ films can be controlled by modification of the microstructure.

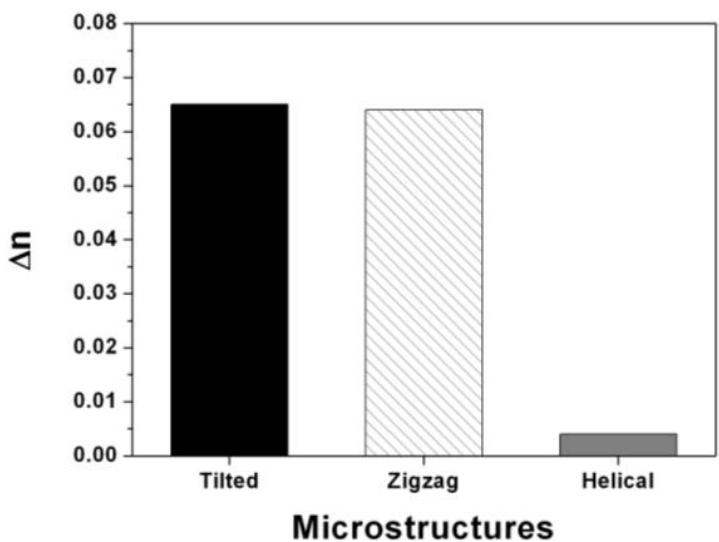

Fig. 7: Optical anisotropy $(\Delta n)$ of $\mathrm{TiO}_{2}$ films with various microstructures at $532 \mathrm{~nm}$

To verify the optical anisotropy of the $\mathrm{TiO}_{2}$ films, transmission intensity of $\mathrm{TiO}_{2}$ films is measured as a function of the rotation angle of the films between two crossed linear polarizers at $532 \mathrm{~nm}$. The transmitted intensity of a phase retardation plate [21] is given by:

$$
I=\frac{I_{0}}{2} \sin ^{2} 2 \theta \sin ^{2} \frac{\Delta \phi}{2}
$$


where $\theta$ is the rotation angle of the film and $\Delta \phi$ is the phase retardation. The relationship between the phase retardation ( $\Delta \phi \square$ ) and the optical anisotropy in the films is given by $\Delta \phi=\frac{2 \pi}{\lambda} d(\Delta n)$ [21]. Where $d$ and $\lambda$ are thickness of the film and wavelength of light, respectively. When the film is optically isotropic $(\Delta n=0)$, the transmitted intensity is zero. On the other hand, if the film is anisotropic $(\Delta n \neq 0)$, the transmitted intensity follows a sinusoidal function of the rotation angle. The measured intensities of the $\mathrm{TiO}_{2}$ film with tilted and zigzag structure at $\alpha=60^{\circ}$ shows maxima at $\theta=45^{\circ}$ and $135^{\circ}$, and minima at $\theta=0,90^{\circ}$, and $180^{\circ}$ respectively, as presented in Fig. 8 (a) and (b). It shows that $\mathrm{TiO}_{2}$ films with tilted and zigzag structures at $\alpha=60^{\circ}$ behave like a sinusoidal function, while the $\mathrm{TiO}_{2}$ film with helical structure at $\alpha=60^{\circ}$ shows a very small and flat intensity, as shown in Fig. 8(c). It shows that $\mathrm{TiO}_{2}$ films with tilted and zigzag structure have a large optical anisotropy, while $\mathrm{TiO}_{2}$ films with helical structure have a very small optical anisotropy.

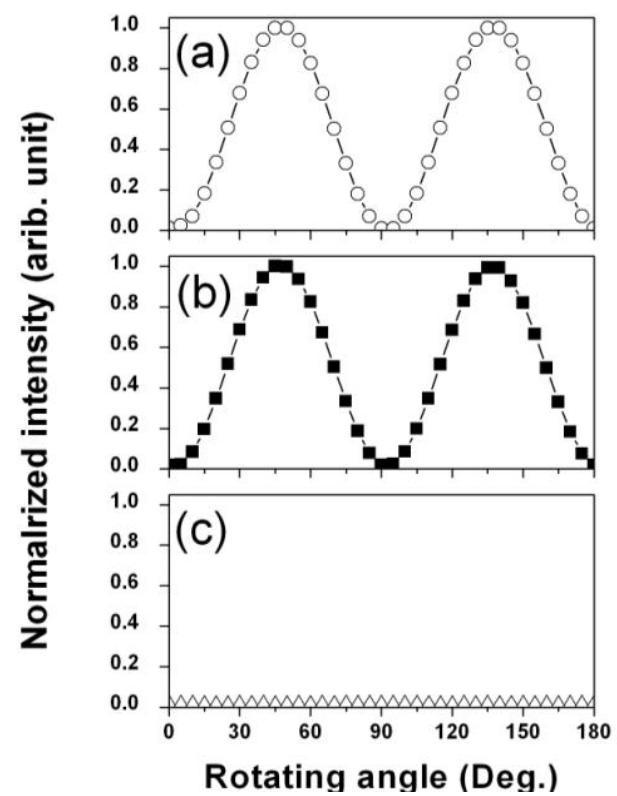

Fig. 8: Normalized transmitted intensity of light at $532 \mathrm{~nm}$ as a function of the sample rotation angle between the two crossed-polarizers

\section{CONCLUSIONS}

Various glancing angles as well as substrate rotation were employed to control the microstructure of $\mathrm{TiO}_{2}$ films using glancing angle deposition (GLAD) and the effects of the microstructure on the optical anisotropy have been studied. The results show that as the glancing angle increases, the column angle and the porosity of $\mathrm{TiO}_{2}$ films increase due to the shadow effects, and the refractive index decreases. The optical anisotropy of the tilted and zigzag structure $\mathrm{TiO}_{2}$ films is maximum at $\alpha=60^{\circ}$, while the $\mathrm{TiO}_{2}$ film of the helical structure appears to experience very small anisotropy, due to the symmetry of the helical structure. It is found that the optical properties (optical anisotropy and refractive index) of the $\mathrm{TiO}_{2}$ films deposited by GLAD, depend on the arrangement of microstructure and can be controlled by modifying the microstructure. 


\section{ACKNOWLEDGEMENT}

This work was supported by the Korea Science and Engineering Foundtion through the Quantum Photonic Science Research Center at Hanyang University.

\section{REFERENCES}

1. Macleod, H.A. (2001), Thin-Film Optical Filters, 3rd ed. (Institute of Physics Publishing, Bristol and Philadelphia, pp. 462-470.

2. Lambrinos, M.F., Valizadeh, R., and Colligon, J.S. (1996), Appl. Opt., vol. 35, pp. 36203626.

3. Gu, P.-F., Chen, Y.M., Hu, X.Q., and Tang, J.-F. (1989), Appl. Opt., vol. 28, pp. 33183322.

4. Woo, S.-H. and Hwangbo, C. K. (2006), Appl. Opt., vol. 45, pp. 1447-1455.

5. Messier, R. and Lakhtakia, A. (1999), Mat. Res. Innovat., vol. 2, p. 217.

6. Gisg, D.A., Summers, M.A., and Brett, M.J. (2006), Photonics and NonostrucyuresFundamentals and Applications, vol. 4, pp. 23-29.

7. Hodgkinson, I., Wu, Q.H., Arnold, M., and Blaikie, R. (2001), Microelectronic Engineering, vol. 57-58, pp. 833-836.

8. Robbie, K. and Brett, M. (1997), J. Vac. Sci. Technol., vol. A 15(3), p. 1460.

9. Young, N.O. and Kowal, J. (1959), Nature (London), vol. 183, p. 104.

10. Popta, A.V., Sit, J.C., and Brett, M.J. (2004), Appl. Opt., vol. 43, p. 18.

11. Rovira, P.I., Yarussi, R.A., Collins, R.W., Venugopal, V.C., Lakhtakia, A., Messier, R., Robbie, K., and Breett, M.J. (1998), Thin Solid Films, vol. 313-314, p. 373.

12. Woo, S.-H. and Hwangbo, C.K. (2006), J. Korean Phys. Soc., vol. 48, pp. 1119-1204.

13. Robbie, K., Friedrich, L.J., Dew, S.K., Smy, T., and Brett, M.J. (1995), J. Vac. Sci. Technol., vol. A 13, p. 1032.

14. Beydaghyan, G., Kaminska, K., Brown, T., and Robbie, K. (2004), Appl. Opt., vol. 43, p. 28.

15. Motohiro, T. and Taga, Y. (1998), Appl. Opt., vol. 28, p. 13.

16. Hodgkinson, I., Wu, Q.H., Knight, B., Lakhtakia, A., and Robbie, K. (2000), Appl. Opt., vol. 39, p. 4.

17. Hodgkinson, I., Wu, Q.H., Arnald, M., Silva, L.D., and Blaikie, R. (2004), Curr. Appl. Phys., vol. 4, p. 106.

18. Kaminska, K. and Robbie, K. (2004), Appl. Opt., vol. 43, p. 7.

19. Kennedy, S.R. and Brett, M.J. (1997), J. Vac. Sci. Technol., vol. A 15, p. 1460.

20. Nieuwenhuizen, J.M. and Haanstra, H.B. (1966), Philips Tech. Rev., vol. 27, p. 87.

21. Yeh, P. (1988), Optical waves in Layered Media (John Willy \& Sons, New York), pp. 215232. 